\title{
Neuroscience 2012
}

\section{3-17 OCTOBER 2012}

Ernest N. Morial Convention Center

New Orleans, LA

Approximate attendance: 28,500

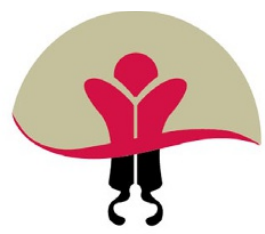

NEUROSCIENCE 2012

Council on the Developing Child, a policy group that informs legislators on research related to brain and childhood development.

New research on brain development during early childhood was introduced at Neuroscience 2012 during a press conference on Tuesday morning. Layla Banihashemi (University of Pittsburgh, PA) presented work showing that physical abuse during early childhood could alter communication between "body control" areas in the brain, potentially predisposing adults to cardiovascular disease and mental health disorders. Eric Pakulak (University of Oregon, Eugene) discussed his research associating childhood poverty with changes in working memory and attention in adults. Suzanne Houston (University of Southern California, Los Angeles) also reported that parental education levels and income are positively correlated with brain size in children. In animal research, Mar Sanchez (Emory University, Atlanta, GA) discussed studies showing that chronic stress in infant rhesus macaques led to changes in stress hormone production and in development of the amygdala that in turn led to increases in fearful and aggressive behaviors.

Neurodegeneration in aging and disease was another focal point at Neuroscience 2012. Lennart Mucke gave a Special Lecture on Tuesday (16 October) on "Alzheimer Mechanisms and Therapeutic Strategies," and several presenters reported new results from studies targeting early identification of the disease. Lori Beason-Held (National Institute on Aging, Bethesda, MD) showed that people with age-related cognitive impairment underwent changes in brain function that could be detected by positron emission tomography years before they developed symptoms. William Klein (Northwestern University, Evanston, IL) presented an antibody-based imaging probe that could distinguish between diseased and normal brain tissue in a mouse model of Alzheimer's disease. Paula Desplats (University of California, San Diego) identified epigenetic signatures of Alzheimer's disease and two other disorders that could aid accurate diagnosis and enable early treatment.

In a press conference on Monday (15 October) publicizing the latest results, moderator Samuel Gandy (Mount Sinai School of Medicine, New York, NY) said, "Being able to detect AD [Alzheimer's disease] early-perhaps even before symptoms begin-is an essential pre-condition if we are to develop effective treatments that slow or stop the changes that occur in the brain during Alzheimer's." 